\title{
Freeze-fracture Shadow-casting (FreSCa) Cryo-SEM as a Tool to Investigate the Wetting of Micro- and Nanoparticles at Liquid-Liquid Interfaces
}

\author{
Lucio Isa $\S^{*}$ \\ §SCS-Metrohm Foundation Award for best oral presentation
}

\begin{abstract}
Since the seminal work of Pickering and Ramsden more than a century ago, adsorption of solid microand nanoparticles at the interface between two fluids has been recognized as a means to enormously improve emulsion stability against coalescence. Despite their long-standing use in a vast range of practical applications, several key issues regarding the behavior of small objects at liquid interfaces still remain unresolved. In particular, current techniques fail to investigate the properties of individual particles smaller than $500 \mathrm{~nm}$. An exception to this scenario is a technique that we have recently developed, based on freeze-fracture cryo-SEM, which for the first time makes it possible to measure the wetting properties of single nanoscale objects through a metal shadow-casting protocol. In this work we present additional details and results which showcase the potential of this novel tool as the benchmark for in situ characterization of particles at interfaces.
\end{abstract}

Keywords: Freeze-fracture cryo-SEM · Liquid interfaces · Microparticles and Nanoparticles · Wetting

\section{Introduction}

Micro- and nanoparticles adsorbed at the interface between two liquids find multiple uses in a large host of practical applications. Investigating their properties in situ at the interface is therefore highly relevant in order to control and direct their employment. In this article, we present additional details of a technique that we have recently developed ${ }^{[1]}$ to measure the wetting properties of micro- and nanoscale objects in situ at a liquid-liquid interface. Our approach offers superior resolution, capabilities and flexibility compared to other state-of-the-art methods.

Liquid (oil-water) interfaces play a paramount role in naturally occurring materials and are of key importance in a vast range of industrial products. Often they are found in the form of emulsions, droplets of one liquid dispersed in a second immiscible liquid, which are commonly employed

\footnotetext{
${ }^{*}$ Correspondence: Dr. L. Isa ETH Zürich

Laboratory for Surface Science and Technology

Department of Materials

Wolfgang-Pauli-Strasse 10

$\mathrm{CH}-8093$ Zürich

Tel.: +41446336376

E-mail: lucio.isa@mat.ethz.ch
}

in many consumer products, including drugs, food stuff, cosmetics and chemicals. Emulsions are thermodynamically unstable against coalescence; by merging, two droplets reduce the surface-to-volume ratio of the system, thus reducing the interfacial free energy. This has the practical consequence that, if left undisturbed, emulsions phase-separate into the two pure constituents to minimize the interfacial area. Despite this, the emulsions that we meet in our everyday life, e.g. hand creams or mayonnaise, are frequently long-lived. This stability, unexpected from purely thermodynamic arguments, stems from the fact that those are not simple emulsions; the surface of the droplets is in fact typically coated by an interfacial film of surface-active molecules or solid particles. ${ }^{[2,3]}$ In particular, solid particles are bound to a liquid interface by the fact that by sitting there, they remove interfacial area between the two fluids and thus lower the system's free energy. ${ }^{[4]}$ The corresponding trapping energies can greatly exceed thermal energy even for small particles, yielding virtually irreversible binding. ${ }^{5]}$ In this case, if two droplets are to coalesce, some particles first need to be expelled from the interface, which has a very high energy cost. This implies that the system is trapped in a metastable state and it is kinetically stabilized against coalescence.

The parameter that mostly determines the stability of solid particle adsorption at a liquid interface is the three-phase contact angle. The familiar concept of the macroscopic three-phase contact angle of a liquid on a solid surface surrounded by a gas (or another liquid) can be extended when the solid material is shrunk to the dimensions of a micro- or nanoparticle. In this case, the particle is adsorbed at the interface and its position relative to the interface itself is determined by the balance of the interfacial energies of all the phases, analogously to the contact angle of a liquid onto a solid surface. ${ }^{6]}$ The so-defined contact angle $\theta$ determines which portion of the particle is exposed to which liquid phase and also the cross-section of the particle at the interface. In particular, if the particle is mostly in the oil phase, its contact angle defined from the water is larger than $90^{\circ}$ and the particle is termed hydrophobic. If conversely the particle is mostly exposed to the water, its contact angle is less than $90^{\circ}$ and it is said to be hydrophilic. Particles equally exposed to both phases are neutrally wetting and have a contact angle of $90^{\circ}$. It is now commonly accepted that the wettability of solid particles is responsible for the type of emulsions that can be prepared. Hydrophilic particles give in fact oil-in-water emulsions, while hydrophobic particles yield water-in-oil emulsions. ${ }^{[2]}$ As anticipated, the contact angle has a direct effect on the stability of the emulsion. In fact, the adsorption energy of a single particle is given by $\Delta E=-\pi r^{2} \gamma_{0}(1-|\cos \theta|)^{2}$, 
where $r$ is the particle radius and $\gamma_{0}$ is the interfacial tension between the two pure liquids. ${ }^{[4]}$ Such trapping energy can be of the order of several thousand kT even for small objects $(r \sim 10 \mathrm{~nm})$, if they are neutrally wetting. ${ }^{[5]}$ For particles with more marked hydrophilic or hydrophobic characters, the adsorption energy decays rapidly and can become comparable to thermal energy, leading to reversible adsorption with important consequences on material fabrication. ${ }^{[7]}$

The contact angle $\theta$ has also consequences on how particles interact at a liquid interface. ${ }^{[8]}$ In particular, it has been shown that charged particles interact as electrostatic dipoles at oil-water interfaces. ${ }^{[4]}$ Even though the detailed origin of such dipolar interactions is still under debate, ${ }^{[9]}$ they depend strongly on the contact angle, i.e. to vanish compared to capillary interactions for hydrophilic particles, which are found to aggregate at the interface. ${ }^{[10]}$

Colloidal particles can also be used as probes to measure the rheological properties of surrounding fluids (microrheology). In such technique the thermally driven motion of a colloidal particle is connected to the complex modulus of the surrounding fluid by applying a generalized StokesEinstein (mobility-diffusion) relation. ${ }^{[1]}$ This method is now well established for bulk systems but it can also be extended to liquid interfaces. ${ }^{[12]}$ Compared to standard interfacial rheological tools, using small tracer particles allows minimization of the contributions coming from shearing the surrounding liquid phases. This fact maximizes the contribution from the interface viscoelasticity and thus offers higher sensitivity in relation to larger probes. Knowing the contact angle of the tracers is therefore crucial since the drag coefficient is a direct function of $\theta .{ }^{[13]}$ Additionally, using small probes also offers the possibility to investigate the structure of interfacial layers at the microscale and highlight features such as structural heterogeneity ${ }^{[14]}$ and presence of alignment. [15]

Given the importance of single-particle contact angles, a host of different techniques have been developed to measure $\theta$ of solid particles at liquid interfaces, as reviewed in ref. [1]. Despite the significant advances made in the past two decades, the limiting factor for the available techniques is the impossibility to measure contact angles of individual particles on the nanoscale. In other words, contact angles of individual objects can be directly measured only if the particles are larger than $500 \mathrm{~nm}$ or only average measurements are available for smaller particles. An exception to this scenario is a method that we have recently developed where, by means of freeze-fracture and cryo-SEM, we demonstrated the measurement of contact angles of individual particles as small as $10 \mathrm{~nm}$ in diameter at alkane oils-water interfaces. ${ }^{[1]}$ Such resolution opens up possibilities to investigate objects of true nanotechnological interest, e.g. functionalized nanoparticles for cellular uptake or targeted drug-delivery, where the wetting properties are crucial to cross cell walls. ${ }^{[16]}$

Sample preparation using freeze-fracture consists of ultra-rapid freezing of liquid samples, which are subsequently fractured to expose the internal structure. The technique was originally developed to image biological specimens, where avoiding water crystallization is crucial to preserve structural information. In particular, it was employed to investigate protein and lipid nanostructures on cell membranes ${ }^{[17]}$ and it has now become the standard technique to characterize lipid-water interfaces of isolated systems ${ }^{[18]}$ or within cells. ${ }^{[19]}$ Recent developments of the technique now see it applied to study other soft systems, including drugs, ${ }^{[20]}$ elusive phase diagrams of oil/ water/surfactant systems, ${ }^{[21]}$ direct investigation of liposome formation in microfluidic devices ${ }^{[22]}$ and nanoscale surface wetting. ${ }^{[23]}$ Freeze-fracture is also frequently employed to study emulsions, with the aim to expose the surface of droplets and study the arrangement of adsorbed particles. ${ }^{[24,25]}$

In the next section we will discuss some features of our freeze-fracture, shadow-casting (FreSCa) cryo-SEM method, adding some details compared to the first publication. ${ }^{[1]}$ In the results section new data are presented, with the focus on additional aspects which highlight the flexibility of the method. Finally, the possibilities of further expanding this method to new systems are discussed in the conclusions.

\section{Method: Freeze-fracture, Shadow- casting (FreSCa) Cryo-SEM}

FreSca cryo-SEM experiments are carried in custom-built copper holders which 'sandwich' a particle-laden, planar oil-water interface. Prior to use, the copper holders are cleaned by ultrasonication in $95 \%$ sulphuric acid and subsequently in ethanol for several minutes. Their inner surface is mechanically roughened (scratching with a blade) and the side to be contacted with water is exposed to a negative glow discharge (Emitech K100X, Quorum Technologies Ltd, UK) for 1 minute to render the surface hydrophilic and thus promote adhesion during freezing and fracture. One side of the holder has a $200 \mu \mathrm{m}$ deep cavity which is precisely filled with $0.5 \mu \mathrm{L}$ of the particle suspension, the other side is flat and is used as a lid to close the container. The second liquid $(3.0 \mu \mathrm{L})$ is either carefully placed on the water surface to create the liquid-liquid interface or placed on the flat copper plate and then the holder is closed. Excess liquid is squeezed out when closing and a thin liquid film $(\sim 100 \mu \mathrm{m})$ separates the two sides. Depending on the particle solubility, either the water or the oil phase is placed in the cavity.

The 'sandwich' holder is then clamped and frozen in a liquid propane jet freezer (Bal-Tec/Leica JFD 030, Balzers/Vienna). The cooling rate of $30000 \mathrm{Ks}^{-1}$ is sufficiently high to avoid crystallization and vitrify water upon freezing so to fix the position of the particles at interface as they were in liquid state. After freezing, the samples are mounted onto a double fracture cryo-stage under liquid nitrogen and transferred under inert gas in a cryo-high vacuum airlock $\left(<5 \times 10^{-7}\right.$ mbar Bal-Tec/Leica VCT010) to a pre-cooled freeze-fracture device at -140 ${ }^{\circ} \mathrm{C}$ (Bal-Tec/Leica BAF060 device). The samples are then fractured and partially freeze-dried at $-100{ }^{\circ} \mathrm{C}$ for $1-3$ minutes to remove deposited residual water condensation and ice crystals. This step is followed by unidirectional tungsten deposition at an elevation angle $\alpha=30^{\circ}$ (or $45^{\circ}$ ) to a total thickness $\delta=2-3 \mathrm{~nm}$ at $-120^{\circ} \mathrm{C}$ and by additional $2 \mathrm{~nm}$ with a continuously varying angle between $90^{\circ}$ and $30^{\circ}\left(45^{\circ}\right)$. The first metal deposition creates a shadow behind each of the features protruding from the frozen, fractured interface. The second deposition is instead needed to avoid charging of the shadow during imaging, which may compromise image stability at high magnifications. Freeze-fractured and metal-coated samples are finally transferred under high vacuum $\left(<5 \times 10^{-7} \mathrm{mbar}\right)$ at -120 ${ }^{\circ} \mathrm{C}$ to a pre-cooled $\left(-120{ }^{\circ} \mathrm{C}\right)$ cryo-SEM (Zeiss Gemini 1530, Oberkochen) for imaging either with an in-lens or secondary electron detector.

The outcome of such a preparation protocol is a sample where both the in-plane interface microstructure and the vertical position of each particle relative to the interface can be measured. Such three-dimensional imaging capability stems from the following principle. In a sundial, an object of known height casts a shadow of well-defined length, from which the angle of the sun on the horizon and thus the time can be calculated. Similarly, FreSCa cryoSEM works as an 'inverse' sundial, where by knowing the shadowing angle and measuring the shadow length one can calculate the height of the object casting the shadow. For a spherical particle of radius $r$, this is directly connected to the contact angle $\theta$ $=\cos ^{-1}(|h-r| / r)$, where $h$ is precisely the protrusion height of the particle from the frozen interface responsible for the shadow casting. The accuracy in measuring the contact angle is then directly related to the resolution of the cryo-SEM images from which $r$ and $h$ can be extracted. This improved resolution compared to the other 
available methods is the key factor in the superior performance of our technique.

From simple geometrical considerations, an object with a slope lower than the shadowing angle cannot cast any shadow outside of its base perimeter. This sets the lower limit for direct contact angle measurement using FreSCa cryo-SEM to $\theta=\alpha$. As discussed in our previous publication, ${ }^{[1]}$ shallower deposition angles produce longer shadows and thus smaller relative errors in measuring $\theta$. The standard angle now used for depositions is $30^{\circ}$ and thus particles with a marked hydrophilic character can be measured. Practically, the limit is set when both the particle diameter at the interface and the shadow length fall below 5 pixels; depending on the particle size and the magnification used, this sets the minimum measurable contact angles. ${ }^{[1]}$ Measuring $\theta$ for hydrophobic particles is simpler, given that the diameter of the particle is exposed and thus directly measurable, while for hydrophilic particles it needs to be inferred from the shadow length and particle cross-section at the interface, assuming spherical curvature (see Fig. 1a,b). Contact angles of hydrophilic particles with $\theta \leq \alpha$ can also be measured by measuring the particle cross-section at the interface. Once more assuming hard, spherical particles, $\theta$ can simply be obtained from $\theta=\sin ^{-1}(r / r)$, where $r_{i}$ is the particle radius at the interface (see Fig. 1c). It is worth noting here that in this case the value of $r$ cannot be directly measured for each individual particle; an average particle radius, measured by another technique, is therefore assumed for all the particles and thus only an average contact angle can be measured, losing the single-particle resolution, similar to the work of Arnaudov et al. [26]

In this case the error on contact angle measurements $\Delta \theta$ is calculated as follows:

$$
\Delta \theta=\frac{1}{r \sqrt{1-\left(r_{i} / r\right)^{2}}} \Delta r_{i}+\frac{1}{\sqrt{1-\left(r_{i} / r\right)^{2}}} \frac{r_{i}}{r^{2}} \Delta r,
$$

where $\Delta r_{\mathrm{i}}= \pm 1$ pixel reflects the accuracy in measuring the particle size at the interface and $\Delta r$ comes from particle polydispersity. The combination of $\theta$ measurement approaches reported here and in ref. [1] allows the coverage of the full range of contact angles. A few examples, as well as additional considerations on the methodology are described in the next section.

\section{Results}

The capability of the method to measure both hydrophilic and hydrophobic particles is exemplified in Fig. 2. The cryo-SEM images show $2.5 \mu \mathrm{m}$ diameter

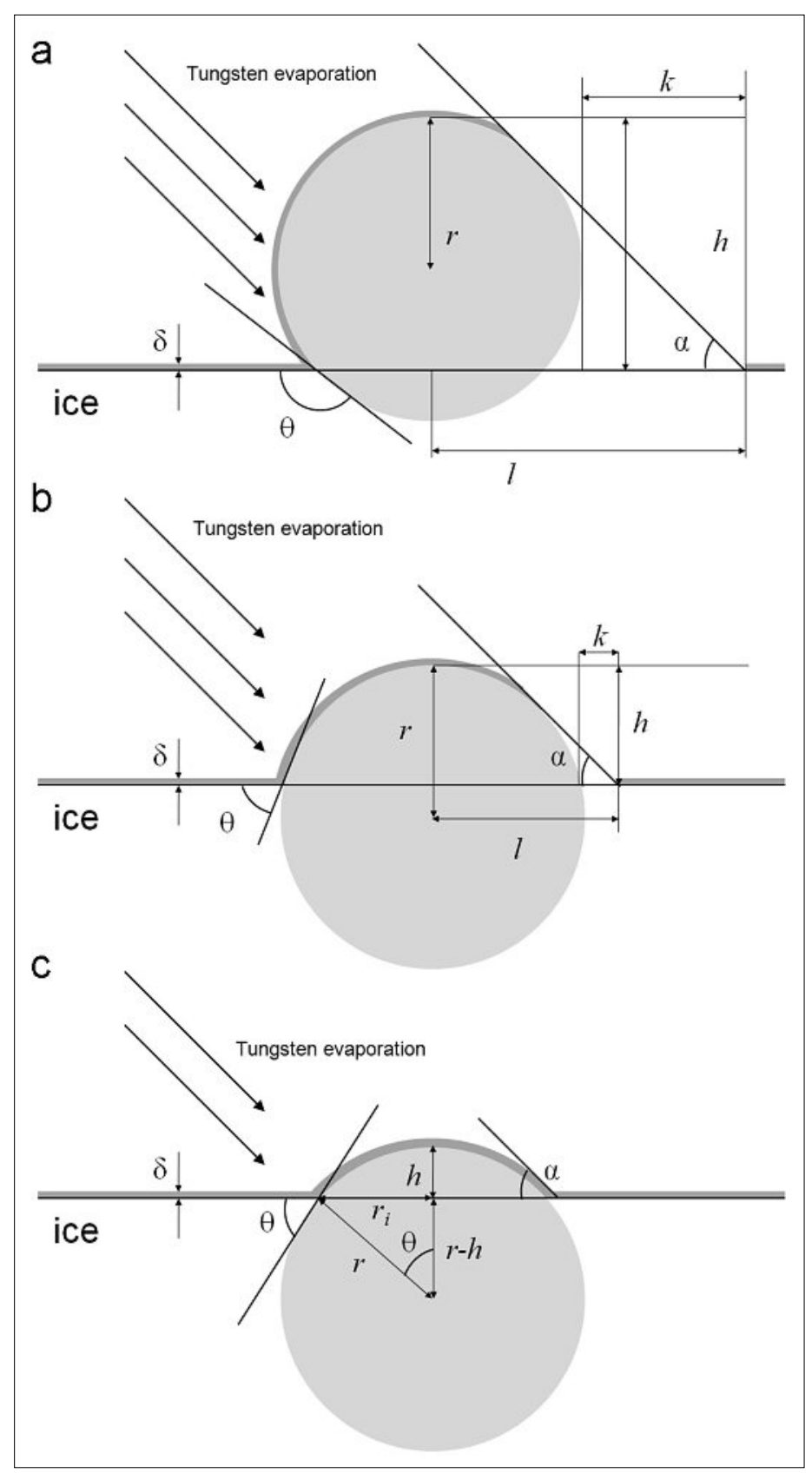

Fig. 1. Schematics of the three possible configurations of a spherical particle embedded in ice after FreSCa. a) Hydrophobic particle, $\theta>\alpha$; b) Hydrophilic particle, $\theta>\alpha$; c) Hydrophilic particle, $\theta \leq \alpha$. The portion of the particle protruding from the ice was initially exposed to the oil phase. For contact angles larger that the deposition angle a shadow is cast and can be used to measure directly both the size and the protrusion height of the particle, and thus its individual contact angle. For contact angles smaller than the deposition angle, no shadow is cast and only the particle size at the interface can be measured; an additional assumption on the particle size is made and thus only average contact angles can be extracted for all particles.

polystyrene latex microspheres (microparticles $\mathrm{GmbH}$ ) at the water/n-decane interface after FreSCa with a shadowing angle $\alpha=45^{\circ}$. The top particles spontaneously adsorbed from the water phase while the ones at the bottom have been spread at the interface using a 4:6 water/isopropyl alcohol mixture. Using an alcohol to promote the formation of a well-controlled particle monolayer at the interface is a commonly used practice ${ }^{[27]}$ but it is evident from the data in Fig. 2 that this has a strong impact on the particle wettability, as previously reported also by other methods. ${ }^{[28]}$ In fact, the presence of the isopropyl alcohol makes the particles more hydrophobic with clear shadows visible behind each of them. In the absence of a spreading solvent the particles are hydrophilic with a contact angle below a value for which no clear shadow (or a shadow shorter than 5 pixels) is observed. The change in wettability has direct consequences if, for instance, the particles are used as tracers for microrheology measurements ${ }^{[12]}$ where it may also affect the interactions between the probe particle and the surrounding interface, e.g. protein or nanoparticle films.

Fig. 3 demonstrates additional features and capabilities of FreSCa cryo-SEM. Starting from Fig. 3a, a high magnification image of $90 \mathrm{~nm}$ amidine (Invitrogen/ Interfacial Dynamics) latex particles immobilized at a water- $n$-decane interface shows that a clear shadow is visible behind each of the particles, corresponding to an average contact angle of $\langle\theta\rangle=$ $124.3^{\circ} \pm 9.5^{\circ}$. This demonstrates the capability of the method to access length scales previously unreachable in terms of single-particle contact angles. In our previous publication we demonstrated that 


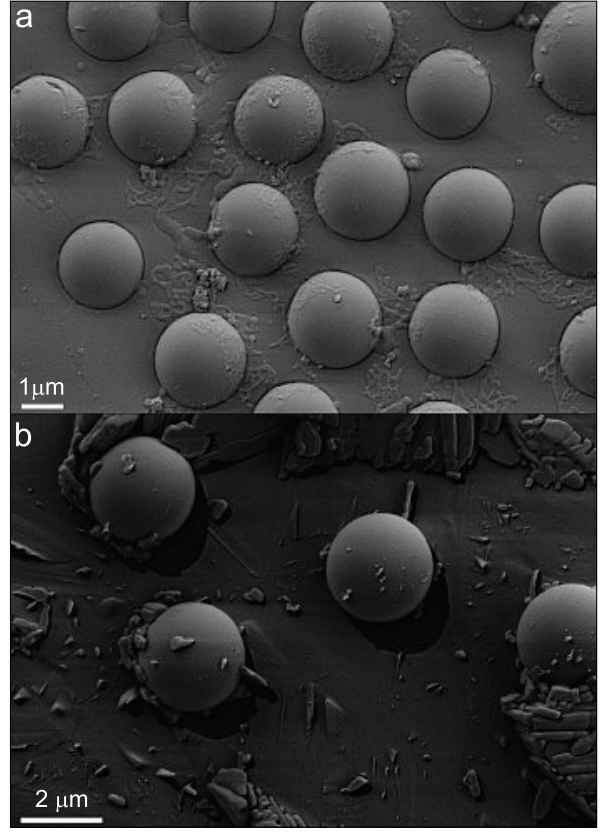

Fig. 2. FreSCa cryo-SEM images of $2.5 \mu \mathrm{m}$ polystyrene latex spheres at the water- $n$ decane interface and shadowing angle $\alpha=$ $45^{\circ}$. The particles in a) are hydrophilic, with a shadow below the 5 pixel limit. An average contact angle is obtained by using the procedure in Fig. 1c $\left(<\theta>=54 \pm 3^{\circ}\right)$. The particles in b) have been assembled adding isopropyl alcohol, often used as a spreading solvent in experiments. Alcohol addition clearly renders the particles more hydrophobic and distinct shadows are visible $\left(<\theta>=106 \pm 6^{\circ}\right)$.

this boundary can be pushed to particles as small as $10 \mathrm{~nm}$ in diameter. ${ }^{[1]}$ Fig. 3b shows that FreSCa cryo-SEM can also be used to examine the microstructure of the interface over large areas and thus to characterize the interactions between particles at the interface. Here we observe a well-defined nearest neighbor distance between the $200 \mathrm{~nm}$ amidine latex particles (Invitrogen/Interfacial Dynamics) at the water- $n$-decane interface driven by dipolar electrostatic interactions ${ }^{[4]}$ and which prevents most particles from aggregating. Large-area in situ inspection is also important to examine interfacial particle assemblies for surface templating. ${ }^{[29]}$ FreSCa cryo-SEM can furthermore be applied to non-planar surfaces, e.g. the surfaces of particle-stabilized emulsion droplets. In conventional freeze-fracture cryo-SEM images of emulsions it is frequently difficult to distinguish between convex and concave surfaces and thus determine clearly if the observed particles are viewed from the oil or the water phase. ${ }^{[25]}$ Fig. $3 \mathrm{c}$ shows the concave surface of an $n$-decane droplet in water after the oil has been removed by the fracture. The curvature of the surface is clearly determined by the fact that the top part is not coated by the tungsten film, due to shadowing from the cavity walls. Effects of surface modifications can also

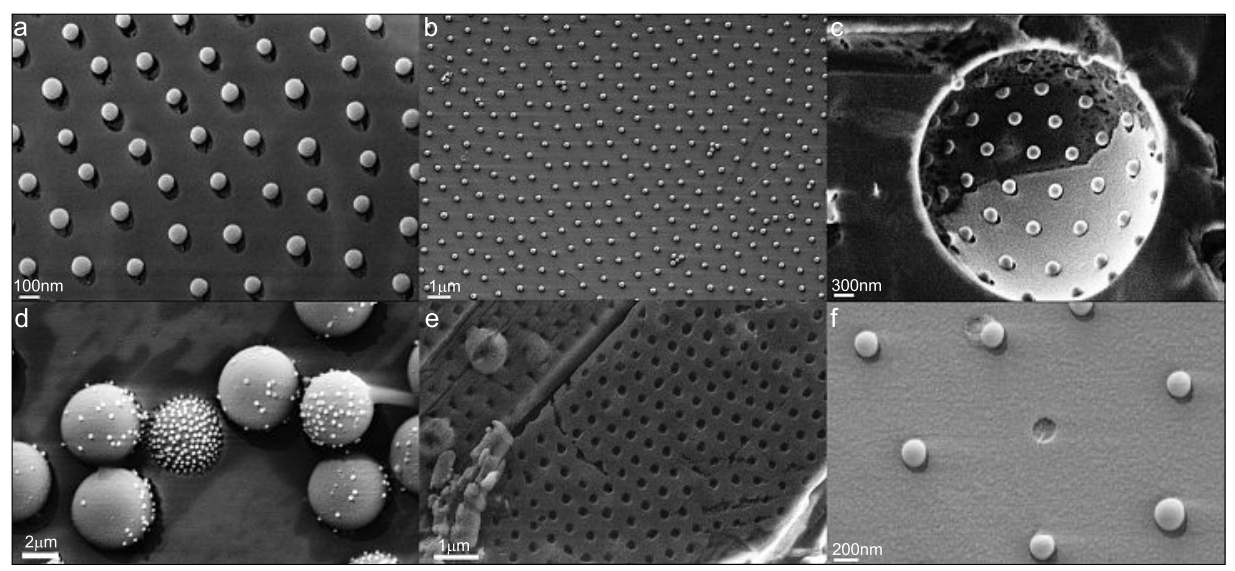

Fig. 3. Examples of FreSCa cryo-SEM images. a) $90 \mathrm{~nm}$ amidine latex particles assembled at a planar water- $n$-decane interface. Shadows from individual particles are clearly visible, underlining the superior resolution of the method compared to other tools. b) $200 \mathrm{~nm}$ amidine latex particles assembled at a planar water- $n$-decane interface. Only limited aggregation is observed and longranged electrostatic dipolar interactions maintain large separation among the particles. c) Surface of an $n$-decane in water droplet after fracture and removal of the oil phase. The $200 \mathrm{~nm}$ amidine latex particles stay trapped at the interface. The upper part of the concave surface is not coated by tungsten due to shadowing from the walls and the latex particles show a regular arrangement due to electrostatic repulsion. d) $500 \mathrm{~nm}$ amidine latex particles coated by $20 \mathrm{~nm}$ citrate gold nanoparticles at a planar water- $n$-decane interface. The gold nanoparticles are hydrophilic and higher surface coatings change the wetting character of the larger latex particles. e) Hollow prints left by $500 \mathrm{~nm}$ amidine latex particles in frozen $n$-decane after freeze-fracture. f) Hydrophobic particles are occasionally ripped out of the ice during the fracture, as demonstrated here for a $200 \mathrm{~nm}$ amidine latex particle at the water- $n$-decane interface.

be directly observed in a single image, as for instance highlighted in Fig. 3d. Here positively charged and hydrophobic 500 $\mathrm{nm}$ amidine latex particles (Invitrogen/ Interfacial Dynamics) are covered by different amounts of negatively charged, hydrophilic $20 \mathrm{~nm}$ citrate gold nanoparticles (BBI International). Different degrees of surface coverage have an impact on the wettability of the large particles, with higher coverage yielding lower contact angles. Large, and hydrophobic enough, particles also leave clearly visible hollow prints in the frozen oil after freeze-fracture, as seen in Fig. 3e. This raises a point which has been frequently at the centre of discussion when presenting data from FreSCa cryo-SEM. All the displayed images always show particles embedded in the vitrified water, even for hydrophobic particles, which should at first thought stay embedded in the oil phase. This is more clearly exemplified in Fig. 3f. Here hydrophobic $200 \mathrm{~nm}$ amidine latex particles at a planar water- $n$-decane interface $(<\theta>=$ $\left.102.4^{\circ} \pm 3.8^{\circ}\right)$ are exposed and imaged after FreSCa cryo-SEM. Despite the hydrophobic character most of the particles stay embedded in the ice after fracture, but we observe that the central particle is ripped out during fracture, leaving a hollow print in the ice.

This phenomenon occurs more clearly and more frequently with larger and even more hydrophobic particles. Fig. 4a in fact shows highly hydrophobic PMMA parti$\operatorname{cles}\left(2.8 \mu\right.$ m diameter, $\left\langle\theta>=129.8^{\circ} \pm 11.8^{\circ}\right)$ still embedded in $n$-decane after fracture. Similarly, in Fig. 4b, other PMMA microparticles are imaged in a region where the fracture did not happen right at the interfacial plane, but a thin layer of frozen oil was left where some of the particles are tightly embedded. On the left side of the image, the water surface is visible and hollow prints are present where particles have been removed during fracture. The particles at the edge of the frozen oil and yet anchored to the ice show long shadows, i.e. hydrophobic character. This effect is even more evident in Fig. 4c, where another region of the same sample in Fig. 4a is visualized. Here most of the particles stayed on the oil side during fracture, leaving hollow prints in the ice, but a few of them were instead sufficiently strongly bound to the ice to be left on the water side and be used for contact angle measurements. We hypothesize that such strong binding, even for highly hydrophobic particles is a direct consequence of the unique expansion of water during freezing. The propane jet freezer is fast enough to prevent water crystallization and thus the corresponding volume changes. Nonetheless, if any structural changes (not observable under the SEM) take place in the water during freezing, these will have the consequence of expanding the water around the particle and thus 'grabbing it' or blocking into place. On the other hand, oils have a standard freezing behavior and shrink when solidifying. This implies that during freezing they can slightly retract from the particles 


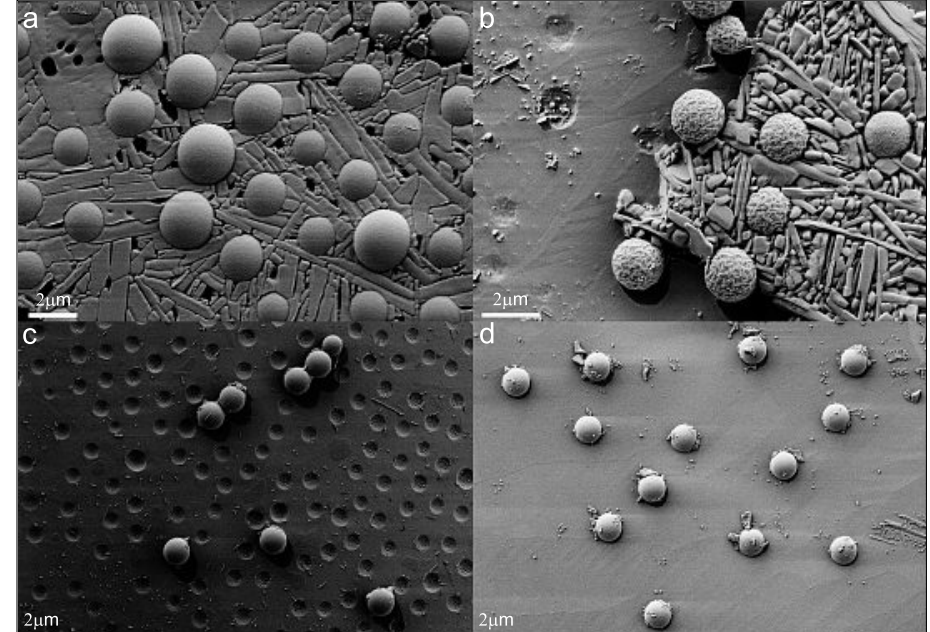

Fig. 4. a) Hydrophobic PMMA microparticles embedded in $n$-decane after freeze fracture. b) Another image of hydrophobic PMMA particles (with rough surfaces) where some of them are embedded in frozen $n$-decane after fracture (right side of the images). On the left side of the image hollow, shallow prints left by the particles on the ice surface are visible. c) Most of the hydrophobic PMMA microparticles assembled at a water/n-decane interface are ripped out of the ice during fracture, leaving visible hollow prints. Only a few were sufficiently strongly trapped to stay on the water side for contact angle measurements. d) For less hydrophobic particles, such as the polystyrene latex microparticles shown here at a water/n-decane interface, some residual frozen oil can be trapped around the contact line during fracture (see debris around the particles' contour). This does not happen for smaller colloids.

and 'let go', making it possible even for hydrophobic objects to stay on the water side after fracture. This is also confirmed by the fact for less hydrophobic particles, such as the $2.8 \mu \mathrm{m}$ diameter polystyrene particles (microparticles $\mathrm{GmbH}$ ) at a water- $n$-decane interface in Fig. $4 \mathrm{~d}(\langle\theta\rangle=$ $85.0^{\circ} \pm 5.2^{\circ}$ ), debris of frozen oil can be left around the particle contour after fracture. Adhesion of sub-micrometer hydrophobic particles to the oil seems to be much less pronounced[30] due to the fact that small volume changes in the frozen oil have much larger consequences on the forces applied to small objects which are more effectively trapped in the vitrified water (see Fig. 3f).

\section{Conclusions}

This article describes additional details of a recently published methodology to measure directly and in situ the contact angles of individual micro and nanoparticles at planar liquid-liquid interfaces. ${ }^{[1]}$ The method is based on freeze-fracture, shadow-casting (FreSCa) cryo-SEM and offers superior resolution compared to other state-of-the-art techniques, making it possible for the first time to measure the contact angles of particles as small as 10 $\mathrm{nm}$ in diameter. In addition to the resolution, the flexibility of the technique has a large impact in tackling problems previously not accessible. The fact that particles are immobilized at the interface by ultra-rapid freezing offers the possibility to study the effect of changing the envi-

\section{Acknowledgements}

The author thanks the Electron Microscopy Centre at ETH Zurich (EMEZ) and Prof. N. D. Spencer for support. The PMMA particles were provided by Dr. A Schofield at the University of Edinburgh. This work was supported by the SNSF Ambizione Fellowship PZ00P2_142532/1.

Received: February 28, 2013

[1] L. Isa, L. Falk, R. Wepf, E. Reimhult, Nat. Comms. 2011, 2, 438.

[2] 'Colloidal particles at liquid interfaces', Eds. B. P. Binks, T. S. Horozov, Cambridge University Press, Cambridge, 2006.

[3] E. Dickinson, Trends Food Sci. Technol. 2012, $24,4$.

[4] P. Pieranski, Phys. Rev. Lett. 1980, 45, 569.

[5] B. P. Binks, S. O. Lumsdon, Langmuir 2000, 16,8622 .

[6] R. Aveyard, J. H. Clint, J . Chem. Soc., Faraday Trans 1996, 92, 85

[7] Y. Lin, H. Skaff, T. Emrick, A. D. Dinsmore, T. P. Russell, Science 2003, 299, 226.

[8] F. Bresme, M. Oettel, J. Phys.: Condens. Matter 2007, 17, 413101.

[9] K. Masschaele, B. J. Park, E. M. Furst, J. Fransaer, J. Vermant, Phys. Rev. Lett. 2010, 105, 048303 .

[10] T. S. Horozov, R. Aveyard, J. H. Clint, B. P. Binks, Langmuir 2003, 19, 2822.

[11] T. G. Mason, K. Ganesan, J. H. vanZanten, D. Wirtz, S. C. Kuo, Phys. Rev. Lett. 1997, 79, 3282.

[12] F. Ortega, H. Ritacco, R. G. Rubio, Curr. Opin. Colloid Interface Sci. 2010, 15, 237.

[13] T. M. Fischer, P. Dhar, P. Heinig, J. Fluid Mech. 2006, $558,451$.

[14] M. H. Lee, D. H. Reich, K. J. Stebe, R. L. Leheny, Langmuir 2010, 26, 2650.

[15] L. Isa, J.-M. Jung, R. Mezzenga, Soft Matter 2011, 7, 8127 .

[16] A. Verma, F. Stellacci, Small 2010, 6, 12.

[17] H. Moor, K. Muhlethaler, J. Cell Biol. 1963, 17, 609.

[18] H. W. Meyer, W. Richter, Micron 2001, 32, 615.

[19] U. B. Sleytr, A. W. Robards, J. Microsc.-Oxford $1977,111,77$.

[20] V. Klang, C. Valenta, N. B. Matsko, Micron $\mathbf{2 0 1 3}, 44,45$

[21] L. Issman, Y. Talmon, J. Microsc. 2012, 246, 60.

[22] A. Jahn, F. Lucas, R. A. Wepf, P. S. Dittrich, Langmuir 2013, 29, 1717.

[23] K. Rykaczewski, T. Landin, M. L. Walker, J. H. J. Scott, K. K. Varanasi, ACS Nano 2012, 6, 9326.

[24] M. Destribats, V. Lapeyre, M. Wolfs, E. Sellier, F. Leal-Calderon, V. Ravaine, V. Schmitt, Soft Matter 2011, 7, 7689.

[25] S. Schmidt, T. T. Liu, S. Rutten, K. H. Phan, M. Moller, W. Richtering, Langmuir 2011, 27, 9801.

[26] L. N. Arnaudov, O. J. Cayre, M. A. C. Stuart, S. D. Stoyanov, V. N. Paunov, Phys. Chem. Chem. Phys. 2010, 12, 328.

[27] S. Reynaert, P. Moldenaers, J. Vermant, Langmuir 2006, 22, 4936.

[28] A. Maestro, L. J. Bonales, H. Ritacco, R. G. Rubio, F. Ortega, Phys. Chem. Chem. Phys. 2010, 12,14115 .

[29] L. Isa, K. Kumar, M. Müller, J. Grolig, M. Textor, E. Reimhult, ACS Nano 2010, 4, 5665.

[30] J. S. Sander, L. Isa, P. A. Ruhs, P. Fischer, A. R. Studart, Soft Matter 2012, 8, 11471.

[31] V. N. Paunov, Langmuir 2003, 19, 7970.

[32] K. Geisel, L. Isa, W. Richtering, Langmuir 2012, 28, 15770.

[33] D. M. Kaz, R. McGorty, M. Mani, M. P. Brenner, V. N. Manoharan, Nat. Mater. 2012, $11,138$. 